\title{
Transforming Outreach Education: Implementing Industrial Engineering Class- room Activities as Outreach Projects
}

\section{Dia St. John, University of Arkansas}

Dia St. John is the Advanced Manufacturing Program Coordinator at Araphoe Community College and a Ph.D. Candidate in the Department of Industrial Engineering at the University of Arkansas where she also earned her B.S.I.E. and M.S.I.E.

\section{Mr. Eric Specking, University of Arkansas}

Eric Specking serves as the Director of Undergraduate Recruitment for the College of Engineering at the University of Arkansas. He directs the engineering recruitment office, most of the College of Engineering's K-12 outreach programs, and the college's summer programs. Specking is actively involved in the Industrial Engineering and Engineering Management divisions and is the current Chair-elect of the ASEE Diversity Committee. Specking received a B.S. in Computer Engineering and a M.S. in Industrial Engineering from the University of Arkansas and is currently working on a $\mathrm{PhD}$ in Industrial Engineering at the University of Arkansas. 


\title{
Transforming Outreach Education: Implementing Industrial Engineering Classroom Activities as Outreach Projects
}

\begin{abstract}
Outreach is vital to increasing pre-college interest in engineering fields, especially industrial engineering. Most outreach literature, focuses on the recruitment of students, content, and the impact on participants. In 2015, the authors, St. John and Specking, proposed a framework to adapt college-level lessons for outreach activities.[5] This work will 1) discuss a modified version of the 2015 framework that emphasizes implementation, assessment, and continuous improvement, 2) provide an example application that was successfully implemented at multiple sections of a University of Arkansas summer camp with assessment data, and 3) provide an additional example of a previously implemented activity to emphasize the cycle of implementation, assessment, and improvement. The University of Arkansas used pre and post surveys to measure the camp success. This resulted in a $10 \%$ increase in participant interest in industrial engineering, a 50\% increase in participant understanding of industrial engineering, and a $12 \%$ increase in participant excitement for industrial engineering.
\end{abstract}

\section{Introduction}

The National Center for Educational Statistics states that $10.7 \%$ of the 2009 U.S. degrees awarded were in STEM fields.[1] The U.S. News/Raytheon STEM Index demonstrated a recent upward trend in the number of STEM graduates, but a shortage of qualified local STEM applicants for employers still exist.[2] Arkansas had the second lowest percentage of STEM degrees awarded in 2009 (8.5\%), and this percentage fell from 2001 levels by 20.7\%.[1] In Arkansas, the College of Engineering has taken a lead role in addressing this issue, investing in quality programs that prepare the state's K-12 students to proceed into engineering degree curricula. This is a large project due to the state's social economics and students' STEM preparedness. According to the Arkansas Department of Education 2015-2016 data, 61\% of public school students receive free or reduced lunch with $41 \%$ of Arkansas school districts containing populations where at least $70 \%$ of students receive free or reduced lunch (15 districts between 90\% - 100\%).[3] According to ACT, Arkansas students perform lower than the national average on all sections, especially in math (below a 20).[4] This means outreach activities are imperative to help expose, excite, and prepare students to ensure a diverse, high quality pipeline of future engineers.

Outreach can be very challenging to balance teaching engineering concepts and making sure students are enjoying the event. It is particularly challenging to achieve this balance the first time an event is implemented. It is, therefore, important to focus on the continuous improvement of outreach methodologies in order to maximize event effectiveness. Projects must be assessed and reevaluated for possible improvements both to student understanding and overall experience. Industrial engineering further complicates these difficulties since so few students have any preexisting understanding of industrial engineering principles and often have misconceptions.[6] Capobianco et al. found that students asked to picture an engineer describe a mechanic, laborer, or technician.[7] Industrial engineers fit this mold even less than other branches of engineering, making direct and effective industrial engineering outreach projects imperative. 
The importance of outreach has sparked research into the development and assessment of outreach activities. Creative approaches have been developed for improving participant recruitment efforts, including adapting research methodology [8], utilizing undergraduate student teams [9], and the effective use of social media [10]. Moskal et al. demonstrated the broader impacts created through a middle school pre-college outreach program, including scholarly benefits to the university students and professors leading the outreach efforts.[11] Assessment of outreach activities largely focuses on improvements to student learning, rather than directly assessing the effectiveness of an outreach methodology. A 2007 study provides the possible explanation that those working with outreach are less driven to publish than faculty members doing scholarly research because these individuals are often focused on their teaching methods. They found that only $3 \%$ of abstracts pulled from 700 publications were focused on K-12 outreach, and none of the outreach papers were journal articles.[12]

This paper presents a modified framework for adapting in-class assignments for outreach purposes. The framework is introduced in section 2 , followed by a detailed description of two adapted activities. The first project is based on manual piece-picking strategies and demonstrates the framework linearly. The second project teaches students about facility layout and layout efficiency. This project has been previously implemented and is included to show the cycle of implementation, assessment, and improvement included in the proposed framework.

\section{Framework}

Creating an outreach project, even one adapted from an in-class activity, is not always an easy task. In order to successfully orchestrate an outreach event, it is important to understand the objective of the event and the capabilities of the audience. In 2015, St. John and Specking created a five step framework to help adapt engineering labs, demonstrations, or projects into a grade appropriate project for kindergarten to twelfth grade students. [5] They proposed that faculty should: "1) Identify outreach details, 2) Select the topic or lesson for adaptation, 3) Decide what concepts to include, 4) Determine the most effective teaching methodologies, and 5) Make the chosen method interactive.'[5] The major flaw in this framework is the lack of focus on future improvement. It is implied, but it does not demonstrate the need to test, assess, and re-implement. Therefore, figure 1 presents a new outreach project development framework cycle that lays out a simple process to take familiar, classroom assignments or demonstrations and effectively implement them with a younger, less experienced audience. The following sections provide more detail on each step. 


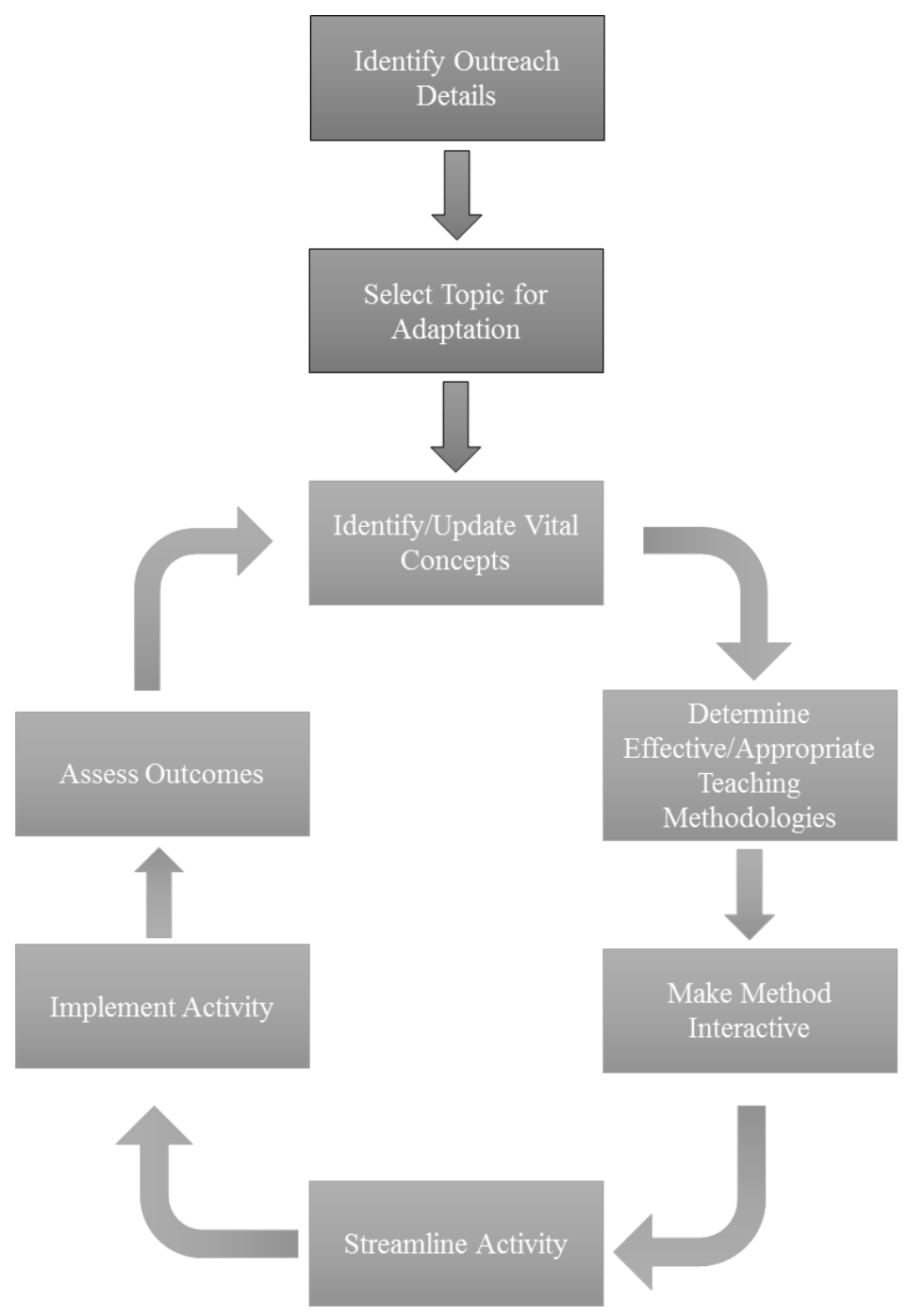

Figure 1: Project Adaptation Framework Cycle

\section{a. Identifying Outreach Details}

Before jumping into activity development, it is important to understand the details of the event. The desired objective(s), location, time frame, budget, number of participants, and participants' age range are all important pieces of information required to shape an effective and appropriate activity.[13] Misunderstanding or misinterpreting any of these details could produce an ineffective activity that squanders resources and provide an inadequate experience for participants. For example, $5^{\text {th }}$ and $6^{\text {th }}$ graders would need more explanation and completion time to successfully complete a project that was designed for $7^{\text {th }}$ and $8^{\text {th }}$ grade students. These participants might become discouraged and negatively connect your activity with the entire field and self-identify as not capable of becoming an engineer.

Event objectives can be determined by you or directed by the organization, depending on the purpose of the project. In-school outreach events are often intended to correspond to current 
course topics and must stay within common core and science standards. Connecting your chosen content to these standards, when possible, will increase your ability to implement the activity in multiple classrooms. In addition, desired objectives can vary widely due to the age of the participants. The same topic could be used in a high school course to introduce specific mathematics concepts but best implemented in a middle school classroom in order to focus on problem solving. On-campus recruitment events offer more freedom in determining activity outcomes and are typically more broadly focused on introducing students to engineering disciplines and careers. It is important that each event is aimed at the prescribed goals.

The location and time frame of the event are important to consider for logistical purposes. Consider the amount of mess an activity will produce as well as the space and setup requirements when matching it with the appropriate facility.[5] These factors are also important to consider when determining the time requirements for a project, including setup and cleanup. The timing of an activity is a very delicate balance. It is important that the activity fits within the allotted time frame, but also that the students do not end up with too much free time upon completing their projects. Project completion time can be incredible difficult to predict. We have seen $5^{\text {th }}$ grade students outperform $9^{\text {th }}$ grade students on the same project. Students' preparedness, problem solving ability, and mindset during the event are often uncertain during event planning and tend to change depending upon the participants' attitudes. One strategy to handle this balance is to pre-divide the activity into manageable pieces. If time remains, challenge the students with one of the extension pieces in order to fill the time without leaving the project feeling unfinished.

The outreach event's budget is another vital consideration. Determine both the total cost and the cost per student before committing to a specific activity. If money is tight, consider activities with class pools of materials rather than a specific list per student. Using this strategy, students must design before requesting their desired supplies. A useful strategy with material pools is to assign a purchasing value to each material and provide a set number of tickets to each group of students to buy the materials from you. This adds the possibility of including additional curriculum, such as performing an economic analysis. It also challenges students to solve a problem with any of the available supplies and encourages innovation. For example, some students will inevitably avoid the more expensive items and build an entire prototype using more simplistic supplies, such as paperclips and rubber bands. These types of activities are also a great way to utilize leftover supplies from previous projects. We have often included random leftover supplies just to see if and how the participants would use them.

The number of participants often influences the location, time frame, budget, and number of facilitators or mentors. Implementing a project for 20 participants is different than for 100. You would need a larger room, budget, and possibly more mentors to help answer questions and keep participants on task. It is possible to stay within the time frame no matter the number of students. It will depend upon the facilitator's comfort level with large groups and ability to manage the classroom and distribute supplies quickly. A useful strategy is to test the project on smaller groups and scale up the project in future iterations of the project adaptation framework cycle.

The age range of the student audience is vital in implementing a successful outreach project. If the activity is too easy, students will get bored. If the activity is too difficult, students will get 
frustrated. It is important when approaching outreach activities to combat the stereotype that becoming an engineer is an impossible task. Participants should instead see how engineers make a difference in society by turning ideas into reality using math, science, and problem solving. Participants should also be reminded that this project dose not capture the entire field being presented. We have found that students tend to connect their success/failures within the project to their ability to work in that field.

\section{b. Selecting the topic or lesson for adaptation}

Our previous work provides activity examples for the three levels of classroom adaptation: lessons that already have an interactive component, lessons that have some portion that can be easily made interactive, and lessons without an interactive component.[5] It is important that the chosen topic can be simplified for the relevant audience skill level, and the best topics can incorporate more than one industrial engineering topic.

\section{c. Identifying the vital concepts for inclusion}

Outreach activities require significantly less depth than a classroom activity. It is important to identify the key pieces of information and sidestep any unnecessary details that could lead to student confusion. It is helpful to remember during this step that the purpose of a classroom activity, to teach a topic to college students that they can apply in industry, is significantly different than the typical purpose of an outreach project, to have fun and get students excited and/or prepared for engineering. Cutting out complicated mathematical concepts is vital to the success of the event, unless your targeted audience is a high-level mathematics course. This is also the easiest place to consider any other industrial engineering topic that can be incorporated. The key here is balancing necessary background information with what is needed to perform the task. You want the participants to be successful.

Facilitators also have to consider the objectives. The amount of depth that is needed will vary depending upon if the objective is to excite, expose, prepare, or do a combination. Preparedness requires more thought and connection to their curriculum. The University of Arkansas College of Engineering has found that a student's math preparedness effects their success in engineering. This means that events that focuses on preparedness needs to reinforce and further develop the participants' math and science skills. The excitement and exposure objectives are the easiest to prepare by ensuring that the activity is fun and interactive but informative.

\section{d. Determining the most effective and appropriate teaching methodologies}

Outreach activities can range broadly in structure. Potential options include presentation, project, demonstration, discussion, or a combination of the four. A presentation is typically required to provide background information and setup the remaining activities. Projects are typically good for engineering outreach events, but demonstration and discussion can be the right choice particularly if the time available is too short for a student project or an interesting demonstration is too expensive or dangerous for students to take part directly. Projects are typically preferred over demonstrations because it provides a more interactive experience. If demonstrations are used, the facilitator should consider how to include more interactions with the entire audience. 
Selecting an assistant from the audience has a great impact on that one individual but does not provide the same experience to the rest of the group. Discussions should be used along with any of the other strategies. Education research suggest that students should talk to their neighbors or in groups about their ideas.[14], [15] Discussions are a fantastic way to end the event by calling on participants to talk about what they learned and what did or did not work. This is also the time to clarify any misunderstandings, answer questions, and reemphasize the connection of the project and objective(s). Most of the time, facilitators will want to use a combination of a presentation, project, demonstration, or discussion.

\section{e. Making the chosen method interactive}

The best outreach activities include hands-on tasks. Open-ended projects that allow students to apply problem solving methods are particularly popular. These hands-on, open-ended projects allow the participants to think for themselves and work through the engineering design loop. This increases their direct interaction and keeps their attention longer. You can even use this as a way to help manage time by encouraging students to continue to develop or improve their solution. Project results are most easily improved through the application of student competition. Students thrive on competition, and giving out prizes can help maintain engagement throughout an activity. The idea of winning something, like a college branded t-shirt, pushes the students to work harder. This also helps with exposure for your institution. Facilitators should be careful on using this motivation technique because it assumes that everyone is motivated by the same things. A competition or a prize will not motivate every student.

\section{f. Streamlining the activity as much as possible}

This piece of the framework is one of the two primary additions to our previous work. After the activity has been designed, but before it has been implemented, it is important to take a step back and think through the details. Streamline any part of the activity that is not absolutely necessary. Any potentially confusing details should be removed or rethought along with any math that does not relate directly to the topic at hand. Use undergraduate students, family members, other faculty and staff, or anyone that you can find to test and discuss your plans. Students especially can provide a perspective to help with the streamlining process. A good example of streamlining is provided with the activity in section 4.

\section{g. Implementing the activity}

At this point, it is time to implement the activity as it has been developed. Make sure to have extensions or additional activities ready in case the project takes less time than expected. The first time an activity is implemented, it is nearly impossible to predict exactly how it will go. Be prepared for things to go wrong and have as many backup plans as possible. The amount of planning performed during the previous steps will impact the implementation. The first six steps are where most of your time should be spent. This will ensure that the implemented activity has the greatest impact.

h. Assessing the outcome 
After the activity has been tested, think critically about what pieces were successful and which pieces failed. Did students all struggle with one segment of the project? Could better materials have been provided? Did you have way too much or way too little of any supplies? How did it fit within the time frame? How did the wrap-up discussion go? Did the students meet your expectations? Ask other staff and even the participants for their perspectives and feedback on the activity. Facilitators can design a formal assessment methodology or empirically determine the results depending upon need. The key is to connect the actual outcomes to the original objectives. For example, ask participants if they had fun and whether they learned something since these are typically important goals for outreach events. It is usually easy to evaluate these objectives throughout the event through observation, but asking the questions directly helps formally assess the activity.

\section{i. Reviewing activities for future improvement}

The second major addition to our previous work is the cyclical nature of activity development. It is important to assess, learn, and adjust after every time the project is used. Use your assessment to guide which pieces of the project should be changed or redeveloped. There is no reason to keep putting your students or your staff through repeated implementation of an activity that has major flaws. Don't be afraid to "go back to the drawing board", especially if the topic is an important one. Be sure to include any other stakeholders in your discussion of potential improvements.

\section{Exercise 1 - Applying the Framework}

The first activity example demonstrates utilizing the framework cycle for the first time. The activity was utilized in a graduate-level course to introduce the concepts of manual piece picking. The in-class activity was very interactive but included many complicated elements that would have overcomplicated an outreach activity.

\section{a. In-class Activity}

A piece picking line was simulated using fifteen numbered cups with straws in each of them. Orders sheets were provided that dictated how many straws were "ordered" from each cup. In order to properly simulate a variety of piece picking strategies, the line needed to have a slow, medium, and fast paced worker. Each worker could pick only one straw at a time, but the slow worker was required to use chopsticks, the medium worker used pliers, and the fast worker used his or her hands.

The activity began by running five minute physical simulations of a variety of piece picking strategies in order to compare the number of orders produced: discrete, batch, zone, and bucket brigade. Once the bucket brigade was determined to be the most effective strategy, the class focused on analyzing the bucket brigade system more closely.

This analysis began by timing each of the three workers picking one straw out of each cup in order to determine pick rates. These pick rates were used to determine the theoretical partition points between the workers. Next, a physical simulation was run of the bucket brigade system 
during which two students recorded the point at which each order was passed from worker to the next in order to calculate the actual partition points. These theoretical and actual values were compared in order to validate the optimal partition formula.

\section{b. Development Process}

This activity was very well suited for use as an outreach project; it used a presentation, demonstration, fun, hands-on interactive activity, and a discussion, while exposing students to the field of industrial engineering and industrial engineering concepts. The outreach details were already known for the event since it was selected for a 90-minute portion of the industrial engineering section of the Explore Engineering Program, a weeklong half-day summer camp aimed to expose students to the various engineering disciplines at the University of Arkansas. Piece-picking was selected since a college level activity already existed that was interactive and could be easily relatable to the targeted age group. The rate timing and partition point calculation and comparison were beyond the scope of a 90 minute outreach activity, so the project was adapted to exclude those pieces and incorporate a student competition. The activity was planned to have the materials pre-setup into stations to help streamline the activity.

\section{c. Outreach Activity}

The event started with a quick interactive presentation on what is industrial engineering and what industrial engineers do. A content specific presentation was given to introduce manual piecepicking, the activity, and the various piece-picking policies excluding bucket brigade. Students were then asked to design their own manual piece picking system by working in groups of five. During practice, they were instructed to designate a slow, medium, and fast worker, but they were not told about the chopsticks or pliers. One of the other two students was designated as the replenishment specialist and redistributed straws to make sure no cups ran out. The remaining student was the order manager and counted the number of completed orders for the line. Each group was given their own fifteen cups, straws, and order sheet simulations to practice developing their piece picking strategy. After plenty of practice time, the student groups reset their piece picking lines to get ready for the competition. Right before starting the competition, it was announced that the slow workers would be using chopsticks and the medium workers would be using pliers. This announcement was delayed in order to keep the students from practicing and becoming too fast with the tools. It was also announced that the winning student group, the team that completed the most orders correctly, would be competing with staff members.

After a 5-10 minute order picking competition, the winning team competed with the staff who used the bucket brigade policy, the optimal piece-picking strategy which the participants did not see during the activity introduction. The competing students' backs were placed to the staff team, so they couldn't see the bucket brigade system and make adjustments to their plan. After the second competition was over (and the staff won), the bucket brigade system was explained to the students and a wrap-up discussion was facilitated. This discussion focused on reinforcing the concepts that were discussed at the beginning of the event, such as optimal solutions.

\section{d. Assessment}


This activity was the industrial engineering section of the 2016 University of Arkansas College of Engineering's Explore Engineering Program summer camp. The Explore Engineering Program aimed to spark an early interest in science, technology, engineering, and mathematics (STEM) through the use of hands-on activities. Participants spent each day looking at various engineering fields offered by the University of Arkansas.

This camp uses a University of Arkansas approved Internal Review Board (IRB) protocol for the use of human subjects. A pre and post survey is used to determine the immediate impact of the camp. Participants are given the pre-survey at the beginning of the first day and the post-survey at the end of the last day. It is part of the program and therefore has a near $100 \%$ completion rate. The approved protocol includes the ability to track each response to an individual. This is useful since the overall study is a longevity research project on the impact of summer camps to help develop future STEM students. The students are tracked to determine if they apply, attend, and graduate from the University of Arkansas with a STEM degree. National clearing house data will be used to find information on the participants whom do not attend the University of Arkansas. Researchers are able to examine the number of times a participant attends a camp and if their survey responses change overtime.

The pre and post surveys ask similar questions to easily connect the responses to an individual's application (name, camp date, and camp attended) and to compare the pre and post survey results. The surveys ask participants to describe their interest, level of understanding, and level of excitement for several fields, including industrial engineering. The interest question uses a 5point Likert scale (no interest, minimal interest, moderate interest, strong interest, and very strong interest). The level of understanding question uses a six-point Likert scale (none, poor, fair, good, very good, and excellent). If they have no understanding, participants are able to indicate no understanding on the level of excitement question since it does not make sense to judge their excitement level if they do not know what said field is. This question also includes a none, not very excited, somewhat excited, excited, and very excited options. The rest of the presurvey include questions to determine how the participant heard about the program, and what they expected to gain from attending. The last few post-survey questions ask if the participant was happy that they attended and what did they learn from attending.

The 2016 Explore Engineering Program reached 92 participants in three Arkansas locations: Batesville, Bentonville, and Fayetteville. The industrial engineering activity described in this paper was used at the Batesville and Bentonville locations. The Fayetteville location received the same overview of engineering that included industrial engineering but did not contain an industrial engineering section with an activity. This location was used as a control group. Bentonville and Fayetteville are both located in Northwest Arkansas. Batesville is located in Northeast Arkansas and serves a different social economic population than Northwest Arkansas. Batesville's section was taught by one of the authors, Specking, who is a professional staff member and an industrial engineer, while undergraduate engineering students who were not industrial engineering majors taught Bentonville and Fayetteville. All staff went through background checks, protection of minors training, and specific summer camp training. The specific summer camp training covered teaching methods and all content. The Explore Engineering Program staff implemented every camp project and went through all the concepts. They were encouraged to teach and practice on each other. 
The Batesville section reached 36 participants and had a $100 \%$ response rate on the pre and post surveys. Table 1 shows the Batesville section's data and demonstrates an increase interest, understanding, and excitement in industrial engineering. This section used the manual piecepicking activity and was taught by an industrial engineer.

Table 1: Pre/Post Survey Results for Batesville Section

\begin{tabular}{|c|c|c|}
\hline Question & Pre-survey Mean Score & Post-survey Mean Score \\
\hline Interest & 2.88 & 3.26 \\
\hline Understanding & 2.49 & 4.08 \\
\hline Excitement & 3.67 & 4.17 \\
\hline
\end{tabular}

The Bentonville section reached 18 participants is the other section that used the manual piecepicking activity, and had a $100 \%$ pre-survey response rate and a $94 \%$ post-survey response rate. The lower response rate on the post survey was caused by a participant leaving early on the last day. This section, like the Batesville section, saw an increase from pre to post in interest, understanding, and excitement for industrial engineering, seen in table 2 . This section saw the greatest percent increase in all three categories and in all three sections. This is interesting since it used the activity but was not taught by an industrial engineering student or an industrial engineer. This helps contribute to the authors' thought that undergraduate students are able to impact grade school students better than faculty and staff. The authors have seen this in several events, but this is the first data that implies it. The key is to ensure that the undergraduate students have the proper training and background knowledge to be effective.

Table 2: Pre/Post Survey Results for Bentonville Section

\begin{tabular}{|c|c|c|}
\hline Question & Pre-survey Mean Score & Post-survey Mean Score \\
\hline Interest & 3.11 & 3.59 \\
\hline Understanding & 2.72 & 4.53 \\
\hline Excitement & 3.33 & 4.47 \\
\hline
\end{tabular}

The last section, Fayetteville, reached 38 participants, did not use an industrial engineering activity, and had a $100 \%$ pre-survey response rate and a $95 \%$ post-survey response rate. Even though this section contained an increase in interest, understanding, and excitement for industrial engineering, as seen in table 3, it did not have as large of an increase as the two previous sections that used the designed activity. Table 4 shows that this section had a significantly lower percent increase than the sections that used the activity. This helps demonstrate that the use of a presentation with a small discussion can be effective, but it is not as effective as using a combination of project, presentation, and discussion. The amount of interaction does matter.

Table 3: Pre/Post Survey Results for Fayetteville Section

\begin{tabular}{|c|c|c|}
\hline Question & Pre-survey Mean Score & Post-survey Mean Score \\
\hline Interest & 3.02 & 3.33 \\
\hline Understanding & 2.66 & 3.99 \\
\hline Excitement & 3.68 & 4.12 \\
\hline
\end{tabular}


Table 4: Pre/Post Survey Results for All Sections

\begin{tabular}{|c|c|c|c|}
\hline & \multicolumn{3}{|c|}{ \% Increase in Mean Pre to Post Scores } \\
\hline Section & Batesville & Bentonville & Fayetteville \\
\hline Interest & $13 \%$ & $15 \%$ & $5 \%$ \\
\hline Understanding & $64 \%$ & $67 \%$ & $30 \%$ \\
\hline Excitement & $14 \%$ & $34 \%$ & $1 \%$ \\
\hline
\end{tabular}

Table 5 shows the overall pre and post survey results for all three Explore Engineering Program locations. In general, the Explore Engineering Program helped camp participants have an increased interest, understanding, and excitement for industrial engineering. The 92 participants on the pre-survey indicated a mean response of 3.02 (moderate interest) when asked to describe their interest in industrial engineering. A $10 \%$ increase, a mean of 3.33, was seen from pre to post with regards to interest. A mean of 2.66 (between poor and fair) was the result of the presurvey question on describing the participant's level of understanding of industrial engineering. This category saw the largest overall percent growth, $50 \%$ since participants indicated a mean of 3.99 on the post survey. This mean indication demonstrates an increase in the students understanding level from between poor and fair to good. The last question regards participant's level of excitement for industrial engineering. This question saw a $12 \%$ growth from pre (mean of 3.68 - not very excited to somewhat excited) to post (mean of 4.12 - somewhat excited to excited).

Table 5: Overall Participants' Pre/Post Survey Results

\begin{tabular}{|c|c|c|}
\hline Question & Pre-survey Mean Score & Post-survey Mean Score \\
\hline Interest & 3.02 & 3.33 \\
\hline Understanding & 2.66 & 3.99 \\
\hline Excitement & 3.68 & 4.12 \\
\hline
\end{tabular}

This assessment proved that the objectives of the Explore Engineering Program and the activity were successful since the participants clearly had an increased interest in STEM. This data provided quantitative proof that proved the facilitators' observations. One facilitator said,

The activity went very well. All of the students seemed to be engaged and having fun. My favorite part was the follow up discussion. The students in my section really seemed to have grasped the concepts and overall ideas.

\section{e. Recommendations and Future Work}

If you utilize this activity, make sure to watch for cheating. Since the straws are all the same in each cup, it is sometimes difficult to ensure students are being honest about picking the correct straws. One way to correct this for future implementation, would be to rotate the order manager so that your team's order manager was counting and acting as a quality control for another group's work. This activity has been very popular with students in the past and is appropriate for a wide range of ages. The duration of the practice time and competition can easily be varied in 
order to better fit the scope of the activity and the attention span of the students. Time studies could be added for older groups if time permitted to teach additional concepts.

\section{Exercise 2 - Reapplying the Framework}

This exercise originated in an introductory level industrial engineering course to introduce facility layout and layout efficiency. It was previously adapted from a PowerPoint layout deliverable to a hands-on physical representation in construction paper for an outreach project.[1] It was originally implemented in the 2013 Explore Engineering Program. There were fairly obvious flaws in the construction paper-based project, so the framework was reapplied in a cyclical fashion to improve student outcomes but has not been implemented.

\section{a. In-class Activity}

Students were asked to develop a facility layout for a fictitious company comprised of 14 departments. The activity relationship between departments and required square footage were supplied along with the building dimensions. Students were required to propose a single facility layout, including a proposed expansion to the existing structure, in a PowerPoint presentation, including a scale drawing, calculation of the layout efficiency, and thought process and critique of their final design.

\section{b. Initial Outreach Activity}

Students developed a new layout for a fast food restaurant from supplied square footage requirements and activity relationships for 9 departments. Students were given a cardboard cutout of the building and 9 colors of construction paper to build the restaurant layout on the cardboard. This required the students to calculate square footage and cut to-scale pieces of paper for their final layout design. The students were encouraged to calculate layout efficiency of any intermediate layout designs before creating their final product. The student with the highest layout efficiency was the winner.

\section{c. Redevelopment Process}

This activity was utilized many times in outreach events (2012 and 2013 Explore Engineering Programs), and the same flaws presented themselves several times. The students struggled with calculating square footage, and the students that didn't struggle calculating it, still struggled to cut the construction paper to the intended size and shape. This is a very unfortunate place for students to struggle since it has very little to do with the point of the project: facility layout and layout efficiency calculations. The square footage calculations could be streamlined out of the project entirely by using different materials.

\section{d. Proposed Outreach Activity}

The new version of this activity has not been implemented. The plan is to replace the cardboard and construction paper with Legos. Each student will be given a Lego baseplate instead of a cardboard base and 9 colors of 2 by 2 Lego bricks. Instead of needing to calculate square 
footage, the bricks will be pre-counted so each student has exactly the number of bricks for each department. Other than this change in material, the project will work exactly as it did before. This simple change in materials will effectively eliminate the major problems with this assignment, and therefore let the students focus on the topic at hand without feeling frustration about the square footage calculations.

\section{e. Recommendations and Future Work}

The new version of this facility layout activity needs to be implemented and assessed for additional improvements. The primary drawback to the material change is budgetary. Legos are significantly more expensive than construction paper, but a similar change to the activity could be implemented in a variety of other ways. Students could be given a sheet of paper with the layout footprint on it and small squares of construction paper they could move around on the sheet. An even lower setup version could have students color a version of the layout divided into a grid, where students color the correct number of squares with each color representing a department. The important part of project reassessment is to figure out a way to remove any obvious bottlenecks.

\section{Conclusion}

This paper presented a modified framework for implementing industrial engineering classroom activities as outreach projects based on the authors' previous work.[5] This modification adds a cyclical nature to the process, emphasizing the importance of implementation, assessment, and continuous improvement. In addition to the framework, we presented two activities developed using this methodology. The first activity demonstrates use of the framework from the first step of topic selection. This first activity was implemented, formally assessed, and shown to improve student understanding of industrial engineering. The second activity utilizes the cycle of improvement to show how a good activity can become a great activity.

\section{Future Work}

This paper presents data that demonstrates the effectiveness of an activity to improve student understanding of industrial engineering. In the future, the framework itself should be validated in order to determine the effectiveness of the methodology as a whole. This validation would ideally come through feedback after successful implementation by others. This crowd approach to validation would increase the pool of industrial engineering activities available for outreach events and increase testing and improvement that leads to a perfected outreach project.

Bibliography

[1] S. Ginder and M. Mason, "Postsecondary Awards in Science, Technology, Engineering, and Mathematics, by State: 2001 and 2009. Web Tables. NCES 2011-226.," National Center for Education Statistics, 2011.

[2] A. Neuhauser and L. Cook, "2016 U.S. News/Raytheon STEM Index," 2016. [Online]. Available: http://www.usnews.com/news/articles/2016-05-17/the-new-stem-index-2016. [Accessed: 11-01-2016]. 
[3] "Free and Reduced School Lunch Data." [Online]. Available: http://www.arkansased.gov/divisions/fiscaland-administrative-services/e-rate/free-and-reduced-school-lunch-data. [Accessed: 11-01-2016].

[4] “The Condition of College \& Career Readiness 2016," 2016.

[5] D. St. John and E. Specking, "From College to K-12: Adapting Industrial Engineering Classroom Exercises for Outreach Purposes," in 2015 ASEE Annual Conference \& Exposition, 2015.

[6] E. Specking, P. E. Kirkwood, and L. Yang, "Perceptions and Misconceptions of Industrial Engineering from First-Year Engineering Students," in 2015 ASEE Annual Conference, 2015.

[7] B. M. Capobianco, H. A. Diefes-dux, I. Mena, and J. Weller, "What is an engineer? Implications of elementary school student conceptions for engineering education," Journal of Engineering Education, vol. 100, no. 2, pp. 304-328, 2011.

[8] S. E. Scanga and K. L. Hajek, "Adapting Your Research into Inquiry-Based Lessons for Public Outreach in High School Classrooms: an Answer to the Calls to Action," The Bulletin of the Ecological Society of America, vol. 91, no. 2, pp. 244-256, 2010.

[9] C. Young and A. E. Butterfield, "Effective Engineering Outreach through an Undergraduate Mentoring Team and Module Database," Chemical Engineering Education, vol. 48, no. 1, pp. 31-36, 2014.

[10] N. Abdullah, S. Chu, S. Rajagopal, A. Tung, and Y. Kwong-Man, "Exploring Libraries' Efforts in Inclusion and Outreach Activities Using Social Media," LIBRI, vol. 65, no. 1, pp. 34-47, 2015.

[11] B. M. Moskal, C. Skokan, L. Kosbar, A. Dean, C. Westland, H. Barker, Q. N. Nguyen, and J. Tafoya, "K-12 Outreach: Identifying the Broader Impacts of Four Outreach Projects," Journal of Engineering Education, vol. 96, no. 3, pp. 173-189, 2007.

[12] M. Borrego, "Development of engineering education as a rigorous discipline: A study of the publication patterns of four coalitions," Journal of Engineering Education, vol. 96, no. 1, pp. 5-18, 2007.

[13] E. Specking and R. Almaian, "An Analytic Hierarchy Process Approach to Engineering Outreach Decisions," in IIE Annual Conference. Proceedings, 2013, p. 1078.

[14] S. H. Chapin, C. O'Connor, and N. C. Anderson, Classroom discussions: Using math talk to help students learn, Grades K-6. Math Solutions, 2009.

[15] M. Orsolini and C. Pontecorvo, "Children's talk in classroom discussions," Cognition and instruction, vol. 9, no. 2, pp. 113-136, 1992. 